\title{
Strangelets in Cosmic Rays
}

\author{
Jes Madsen \\ Department of Physics and Astronomy, University of Aarhus, \\ DK-8000 Århus C, Denmark \\ jesm@phys.au.dk
}

\begin{abstract}
The properties of strangelets are reviewed and two experiments searching for them in cosmic rays are described. The prospects for strangelets as ultra-high energy cosmic rays beyond the classical GZK-cutoff are discussed.
\end{abstract}

\section{Bulk quark mattera}

Quark matter composed of $u$ and $d$-quarks is unstable except for 3-quark baryons (otherwise nuclei would decay to quark matter). An additional Fermi sea with a third flavor, $s$, makes it possible to reduce the energy by increasing the spatial density of quarks. The $s$-quark mass is high compared to that of $u$ and $d$, so stability is most likely for low $s$-quark mass. ${ }^{2-5}$

The Fermi momentum, $p_{F i}$, of a non-interacting, massless quark-flavor, $i$, equals the chemical potential, $\mu_{i}$. The number density is $n_{i}=\mu_{i}^{3} / \pi^{2}$, the energy density $\epsilon_{i}=3 \mu_{i}^{4} /\left(4 \pi^{2}\right)$, and the pressure $P_{i}=\mu_{i}^{4} /\left(4 \pi^{2}\right)$. The sum of the quark pressures is balanced by the confining bag pressure, $B ; \sum_{i} P_{i}=B$; the total energy density is $\epsilon=$ $\sum_{i} \epsilon_{i}+B=3 \sum_{i} P_{i}+B=4 B$, and the baryon number density is $n_{B}=\sum_{i} n_{i} / 3$. For a gas of $u$ and $d$-quarks charge neutrality requires $n_{d}=2 n_{u}$, or $\mu_{2} \equiv \mu_{u}=2^{-1 / 3} \mu_{d}$. The corresponding two-flavor quark pressure is $P_{2}=P_{u}+P_{d}=\left(1+2^{4 / 3}\right) \mu_{2}^{4} /\left(4 \pi^{2}\right)=$ $B$, the total energy density $\epsilon_{2}=3 P_{2}+B=4 B$, and the baryon number density $n_{B 2}=\left(n_{u}+n_{d}\right) / 3=\mu_{2}^{3} / \pi^{2}$, giving an energy per baryon of

$$
\epsilon_{2} / n_{B 2}=\left(1+2^{4 / 3}\right)^{3 / 4}\left(4 \pi^{2}\right)^{1 / 4} B^{1 / 4}=6.441 B^{1 / 4} \approx 934 \mathrm{MeV} B_{145}^{1 / 4},
$$

where $B_{145}^{1 / 4} \equiv B^{1 / 4} / 145 \mathrm{MeV} ; 145 \mathrm{MeV}$ being the lowest possible choice that avoids instability of ordinary nuclei.

A three-flavor quark gas is electrically neutral for $n_{u}=n_{d}=n_{s}$, i. e. $\mu_{3} \equiv$ $\mu_{u}=\mu_{d}=\mu_{s}$. For fixed bag constant the three-quark gas should exert the same pressure as the two-quark gas (leaving also the energy density, $\epsilon_{3}=3 P_{3}+B=4 B$, unchanged). That happens when $\mu_{3}=\left[\left(1+2^{4 / 3}\right) / 3\right]^{1 / 4} \mu_{2}$, giving a baryon number density of $n_{B 3}=\mu_{3}^{3} / \pi^{2}=\left[\left(1+2^{4 / 3}\right) / 3\right]^{3 / 4} n_{B 2}$. The energy per baryon is then

$$
\epsilon_{3} / n_{B 3}=3 \mu_{3}=3^{3 / 4}\left(4 \pi^{2}\right)^{1 / 4} B^{1 / 4}=5.714 B^{1 / 4} \approx 829 \mathrm{MeV} B_{145}^{1 / 4} ;
$$

lower than in the two-quark case by a factor $n_{B 2} / n_{B 3}=\left(3 /\left(1+2^{4 / 3}\right)\right)^{3 / 4} \approx 0.89$, so one may gain of order $100 \mathrm{MeV}$ per baryon by introducing an extra flavor.

For massive $s$-quarks weak equilibrium gives $\mu_{s}=\mu_{d}=\mu_{u}+\mu_{e}$, where $\mu_{e}>0$ corresponds to a small but non-zero density of electrons required for local charge

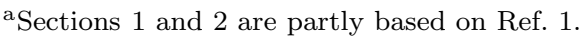


neutrality. For reasonable values of $m_{s}$ of order $100 \mathrm{MeV}$ stable strange quark matter remains possible for a range of $B$.

Quark matter at asymptotically high density has an interesting property called color superconductivity. ${ }^{6,7}$ Even the weakest attraction (and such attractions exist in QCD) leads quarks of different colors and flavors to form pairs, much like Cooper pairs in a superconductor, except that the binding in QCD is caused by a direct attraction channel rather than via indirect phonon interaction. The binding energy of a pair, $\Delta$, can be very large, ranging from a few $\mathrm{MeV}$ to over $100 \mathrm{MeV}$. In general these systems are called color superconductors. If all colors and flavors pair in an equal manner one talks about color-flavor locking.

While color-flavor locking seems generic in the infinite density limit, the properties of strange quark matter at densities of order or somewhat higher than nuclear matter density is at the focus of much current research and discussion. This is the density regime of relevance for strangelets, strange stars, and for quark matter cores in hybrid stars (the analogs of neutron stars if quark matter is metastable so that it forms above a certain density in compact star interiors). An additional binding energy per baryon of approximately $-3 \Delta^{2} / \mu$ is introduced in these systems, meaning that extra binding of $10-100 \mathrm{MeV}$ is not unrealistic, thus significantly increasing the likelihood of absolutely stable strange quark matter and strangelets.

\section{Strangelets}

For $A \ll 10^{7}$ the quark part of strange quark matter is smaller than the Compton wavelength of electrons, so electrons no longer ensure local charge neutrality. Therefore Coulomb effects have to be included, though the fortuitous cancellation of $q_{u}+q_{d}+q_{s}=\frac{2}{3}-\frac{1}{3}-\frac{1}{3}=0$ means that Coulomb energy is much less important for strangelets than for nuclei. In particular strangelets do not fission. For $A<10^{3}$ other finite size effects such as surface tension and curvature have to be taken into account.

Several strangelet searches with relativistic heavy-ion collisions as well as cosmic ray searches have been carried out, and others are planned for the future.$^{8-10}$ Most of these searches are sensitive only to low $A$-values, so it is important to know the properties of small lumps of strange quark matter (strangelets).

Mode-filling for large numbers of quarks in a spherical MIT-bag ${ }^{11}$ was performed for $u d$-systems in Ref. 12, and for strangelets in Refs. 5,13-17. All of these calculations were performed for strong fine-structure constant $\alpha_{s}=0$.

The energy per baryon smoothly approaches the bulk limit for $A \rightarrow \infty$, whereas the energy grows significantly for low $A$. For low $s$-quark mass shells are found for $A=6$ (3 colors and 2 spin orientations per flavor), and less conspicuous ones for $A=18,24,42,54,60,84,102$ etc. As $m_{s}$ increases it becomes more and more favorable to use $u$ and $d$ rather than $s$-quarks, and the "magic numbers" change; for instance the first closed shell is seen for $A=4$ rather than 6 .

Strangelet mode-filling calculations can be modified by inclusion of Coulomb 
energy and zero-point fluctuation energy. The Coulomb energy is generally small. The zero-point energy is normally included as a phenomenological term of the form $-Z_{0} / R$, where fits to light hadron spectra indicate the choice $Z_{0}=1.84 .{ }^{15}$ Roughly half of this phenomenological term is due to center-of-mass motion. The proper choice of $\alpha_{s}$ and $Z_{0}$ is tricky. As discussed by Farhi and $\mathrm{Jaffe}^{5}$ the values are intimately coupled to $B$ and $m_{s}$, and it is not obvious that values deduced from bag model fits to ordinary hadrons are to be preferred in the case of strangelets. This uncertainty may have an important effect for $A<5-10$, but the zero-point energy quickly becomes negligible for increasing $A$ as it decreases like $A^{-1 / 3}$. It means, however, that it is difficult to match strangelet calculations to experimental data concerning ordinary hadrons or limits on the putative $A=2 H$-dibaryon.

Mode-filling calculations give the "correct" results as far as the model can be trusted, but for many applications a global mass-formula analogous to the liquid drop model for nuclei is of great use and also gives further physical insight.

Berger and $\mathrm{Jaffe}^{18}$ made such a detailed analysis within the MIT bag model. They included Coulomb corrections and surface tension effects stemming from the depletion in the surface density of states due to the mass of the strange quark. Both effects were treated as perturbations added to a bulk solution with the surface contribution derived from a multiple reflection expansion. A self-consistent treatment including also the very important curvature energy was given in Refs. 16,19,20.

In the ideal Fermi-gas approximation the energy (mass) of a system composed of quark flavors $i$ is given by

$$
E=\sum_{i}\left(\Omega_{i}+N_{i} \mu_{i}\right)+B V+E_{\text {Coul }} .
$$

Here $\Omega_{i}, N_{i}$ and $\mu_{i}$ denote thermodynamic potentials, total number of quarks, and chemical potentials, respectively. $B$ is the bag constant, $V$ is the bag volume, and $E_{\text {Coul }}$ is the Coulomb energy.

In the multiple reflection expansion framework of Balian and Bloch, ${ }^{21}$ the thermodynamical quantities can be derived from a density of states of the form

$$
\frac{d N_{i}}{d k}=6\left\{\frac{k^{2} V}{2 \pi^{2}}+f_{S}\left(\frac{m_{i}}{k}\right) k S+f_{C}\left(\frac{m_{i}}{k}\right) C+\ldots\right\},
$$

where area $S=\oint d S$ (= $=4 \pi R^{2}$ for a sphere) and extrinsic curvature $C=$ $\oint\left(\frac{1}{R_{1}}+\frac{1}{R_{2}}\right) d S$ ( $=8 \pi R$ for a sphere). Curvature radii are denoted $R_{1}$ and $R_{2}$. For a spherical system $R_{1}=R_{2}=R$. The functions $f_{S}$ and $f_{C}$ are given by ${ }^{16,18}$

$$
\begin{gathered}
f_{S}\left(\frac{m}{k}\right)=-\frac{1}{8 \pi}\left\{1-\left(\frac{2}{\pi}\right) \tan ^{-1} \frac{k}{m}\right\}, \\
f_{C}\left(\frac{m}{k}\right)=\frac{1}{12 \pi^{2}}\left\{1-\frac{3}{2} \frac{k}{m}\left(\frac{\pi}{2}-\tan ^{-1} \frac{k}{m}\right)\right\} .
\end{gathered}
$$


In terms of volume-, surface-, and curvature-densities, $n_{i, V}, n_{i, S}$, and $n_{i, C}$, the number of quarks of flavor $i$ is

$$
N_{i}=\int_{0}^{k_{F i}} \frac{d N_{i}}{d k} d k=n_{i, V} V+n_{i, S} S+n_{i, C} C
$$

with Fermi momentum $k_{F i}=\left(\mu_{i}^{2}-m_{i}^{2}\right)^{1 / 2}$.

The corresponding thermodynamic potentials are related by

$$
\Omega_{i}=\Omega_{i, V} V+\Omega_{i, S} S+\Omega_{i, C} C,
$$

where $\partial \Omega_{i} / \partial \mu_{i}=-N_{i}$, and $\partial \Omega_{i, j} / \partial \mu_{i}=-n_{i, j}$. For massless quarks $\Omega_{i, S}=n_{i, S}=0$; $\Omega_{i, C}=\mu_{i}^{2} / 8 \pi^{2} ; n_{i, C}=-\mu_{i} / 4 \pi^{2}$.

Minimizing the total energy at fixed $N_{i}$ gives the pressure equilibrium constraint

$$
B=-\sum_{i} \Omega_{i, V}-\frac{2}{R} \sum_{i} \Omega_{i, S}-\frac{2}{R^{2}} \sum_{i} \Omega_{i, C} .
$$

The optimal composition for fixed baryon number, $A$, can be found by minimizing the energy with respect to $N_{i}$ giving

$$
0=\sum_{i}\left(\mu_{i}+\frac{\partial E_{\text {Coul }}}{\partial N_{i}}\right) d N_{i}
$$

For uncharged bulk quark matter one arrives at the usual energy per baryon

$$
\epsilon^{0}=A^{-1} \sum_{i} N_{i}^{0} \mu_{i}^{0}
$$

where superscript 0 denotes bulk values. The energy minimization, Eq. (9), corresponds to

$$
B=-\sum_{i} \Omega_{i, V}^{0}=\sum_{i} \frac{\left(\mu_{i}^{0}\right)^{4}}{4 \pi^{2}}
$$

where the last equality assumes massless quarks. In the bulk limit the baryon number density is given by

$$
n_{A}^{0}=\frac{1}{3} \sum_{i} \frac{\left(\mu_{i}^{0}\right)^{3}}{\pi^{2}}
$$

and one may define a bulk radius per baryon as

$$
R^{0}=\left(3 / 4 \pi n_{A}^{0}\right)^{1 / 3}
$$

For quark matter composed of massless $u, d$, and $s$-quarks, the Coulomb energy vanishes at equal number densities due to the fact that the sum of the quark charges is zero. Thus it is energetically most favorable to have equal chemical potentials for the three flavors. From the equations above one may derive the following bulk expressions for 3-flavor quark matter:

$$
\mu_{i}^{0}=\left(\frac{4 \pi^{2} B}{3}\right)^{1 / 4}=1.905 B^{1 / 4}=276.2 \mathrm{MeV} B_{145}^{1 / 4}
$$




$$
\begin{gathered}
n_{A}^{0}=\left(\mu_{i}^{0}\right)^{3} / \pi^{2}=0.700 B^{3 / 4} \\
R^{0}=\left(3 / 4 \pi n_{A}^{0}\right)^{1 / 3}=0.699 B^{-1 / 4} .
\end{gathered}
$$

And the energy per baryon is

$$
\epsilon^{0}=3 \mu_{i}^{0}=5.714 B^{1 / 4}
$$

in agreement with Eq. (2).

To first order one may regard Coulomb, surface, and curvature energies as perturbations on top of the bulk solution. ${ }^{18}$ In this approach one gets a strangelet mass $M(=E)$

$$
\begin{aligned}
M & =\epsilon^{0} A+\sum_{i} \Omega_{i, C}^{0} C^{0}=\epsilon^{0} A+\frac{3^{13 / 12} B^{1 / 4} A^{1 / 3}}{\pi^{1 / 6} 2^{1 / 6}} \\
& \approx\left[829 A \mathrm{MeV}+351 \mathrm{MeV} A^{1 / 3}\right] B_{145}^{1 / 4} .
\end{aligned}
$$

For $m_{s}>0$ and $\lambda \equiv m_{s} / \mu_{s}$ the energy minimization, Eq. (12), changes to

$$
B=\sum_{i=u, d} \frac{\left(\mu_{i}^{0}\right)^{4}}{4 \pi^{2}}+\frac{\left(\mu_{s}^{0}\right)^{4}}{4 \pi^{2}}\left[\left(1-\lambda^{2}\right)^{1 / 2}\left(1-\frac{5}{2} \lambda^{2}\right)+\frac{3}{2} \lambda^{4} \ln \frac{1+\left(1-\lambda^{2}\right)^{1 / 2}}{\lambda}\right],
$$

and the baryon number density is now given by

$$
n_{A}^{0}=\frac{1}{3}\left[\sum_{i=u, d} \frac{\left(\mu_{i}^{0}\right)^{3}}{\pi^{2}}+\frac{\left(\mu_{s}^{0}\right)^{3}}{\pi^{2}}\left(1-\lambda^{2}\right)^{3 / 2}\right] .
$$

A bulk radius per baryon is still defined by Eq. (14).

In bulk equilibrium the chemical potentials of the three quark flavors are equal, $\mu_{u}^{0}=\mu_{d}^{0}=\mu_{s}^{0} \equiv \mu^{0}=\epsilon^{0} / 3$. Neglecting Coulomb energy one may approximate the mass of small strangelets as a sum of bulk, surface and curvature terms, using the chemical potential calculated in bulk:

$$
M=\epsilon^{0} A+\sum_{i} \Omega_{i, S}^{0} S^{0}+\sum_{i} \Omega_{i, C}^{0} C^{0},
$$

where $S^{0}=4 \pi\left(R^{0}\right)^{2} A^{2 / 3}$ and $C^{0}=8 \pi\left(R^{0}\right) A^{1 / 3}$. Masses in $\mathrm{MeV}$ for $B^{1 / 4}=145 \mathrm{MeV}$ are (with $s$-quark mass in $\mathrm{MeV}$ given in parenthesis)

$$
\begin{aligned}
M(0) & =829 A+0 A^{2 / 3}+351 A^{1 / 3} \\
M(50) & =835 A+61 A^{2 / 3}+277 A^{1 / 3} \\
M(150) & =874 A+77 A^{2 / 3}+232 A^{1 / 3} \\
M(200) & =896 A+53 A^{2 / 3}+242 A^{1 / 3} \\
M(250) & =911 A+22 A^{2 / 3}+266 A^{1 / 3} \\
M(300) & =917 A+0.3 A^{2 / 3}+295 A^{1 / 3} \\
M(350) & =917 A+0 A^{2 / 3}+296 A^{1 / 3}
\end{aligned}
$$


The lack of a significant Coulomb energy is due to the fortuitous cancellation of charge $+2 e / 3$ up quarks and charge $-e / 3$ down and strange quarks in strange quark matter with equal numbers of the three quark flavors. Because of the non-zero $s$-quark mass the cancellation is not perfect. Typical strangelets have slightly fewer strange quarks compared to up and down, and therefore the net charge is slightly positive. A typical model result (to be compared to $Z \approx 0.5 A$ for nuclei) is ${ }^{22}$

$$
\begin{aligned}
& Z=0.1\left(\frac{m_{s}}{150 \mathrm{MeV}}\right)^{2} A \\
& Z=8\left(\frac{m_{s}}{150 \mathrm{MeV}}\right)^{2} A^{1 / 3}
\end{aligned}
$$

for $A \ll 700$ and $A \gg 700$ respectively (the slower growth for higher $A$ is a consequence of charge screening).

Thus a unique experimental signature of strangelets is an unusually high massto-charge ratio compared to nuclei.

Cooper pairing involves quarks with equal (but opposite) momenta, so the natural ground state of a color-flavor locked system has equal Fermi momenta for up, down, and strange quarks and therefore equal number densities. Thus the total net quark charge is zero for a bulk system. ${ }^{23}$ A finite strangelet has a surface suppression of massive strange quarks relative to the almost massless ups and downs (massive particle wave functions are suppressed at a surface), so the total charge of a color-flavor locked strangelet is positive and proportional to the surface area: ${ }^{24,25}$

$$
Z=0.3\left(\frac{m_{s}}{150 \mathrm{MeV}}\right) A^{2 / 3}
$$

This phenomenon persists even for very large bags, such as strange stars, so color-flavor locked strange stars also have a positive quark charge. ${ }^{24,26,27}$

Writing $M=\epsilon^{0} A+c_{\text {surf }} A^{2 / 3}+c_{\text {curv }} A^{1 / 3}$, with $c_{\text {surf }} \approx 100 \mathrm{MeV}$ and $c_{\text {curv }} \approx$ $300 \mathrm{MeV}$, the stability condition $M<A m_{n}$ may be written as $A>A_{\min }^{\text {abs }}$, where

$$
A_{\text {min }}^{\text {abs }}=\left(\frac{c_{\text {surf }}+\left[c_{\text {surf }}^{2}+4 c_{\text {curv }}\left(m_{n}-\epsilon^{0}\right)\right]^{1 / 2}}{2\left(m_{n}-\epsilon^{0}\right)}\right)^{3} .
$$

Stability at baryon number 30 requires a bulk binding energy in excess of $65 \mathrm{MeV}$, which is barely within reach in unpaired strange quark matter for $m_{s}>100 \mathrm{MeV}$ if, at the same time, $u d$-quark matter shall be unstable. For color-flavor locked strangelets, stability is more likely. Long-lived metastability with respect to neutron emission is possible if $d E_{\text {curv }} / d A+d E_{\text {surf }} / d A<m_{n}-\epsilon^{0}$, or $A$ larger than

$$
A_{\mathrm{min}}^{\mathrm{meta}}=\left(\frac{c_{\mathrm{surf}}+\left[c_{\mathrm{surf}}^{2}+3 c_{\mathrm{curv}}\left(m_{n}-\epsilon^{0}\right)\right]^{1 / 2}}{3\left(m_{n}-\epsilon^{0}\right)}\right)^{3} .
$$

To have $A_{\min }^{\text {meta }}<30$ requires $m_{n}-\epsilon^{0}>30 \mathrm{MeV}$.

Shell effects can have a stabilizing effect not taken into account in the liquid drop model approach above. As stressed by Gilson and $\mathrm{Jaffe}^{15}$ the fact that the slope of 
$E / A$ versus $A$ becomes very steep near magic numbers can lead to strangelets that are metastable (stable against single baryon emission) even for $\epsilon^{0}>930 \mathrm{MeV}$.

\section{The AMS and LSSS experiments}

Strangelet (meta)stability is a theoretical possibility as demonstrated above, but the existence of small baryon number strangelets is ultimately an experimental issue.

Several experiments have searched for strangelets in cosmic rays. While some interesting events have been found that are consistent with the predictions for strangelets, none of these have been claimed as real discoveries. Whether interpreted as flux limits or as detections these results are consistent with the flux predictions from strange star collisions of a few strangelets per year per square meter per steradian given in Ref. 28 (see also Refs. 29-33). More specifically the integrated flux of mass $A$, charge $Z$ strangelets reaching the inner Solar System was predicted to be ${ }^{28}$

$$
F=2 \times 10^{5} A^{-1.067} Z^{-0.6}
$$

per year per square meter per steradian under the conservative assumption of $10^{-10}$ solar masses per year of strangelets being released in our Galaxy from binary strange star collisions. The major unknown is the mass spectrum of strangelets released, but it seems plausible within quite different scenarios that some strangelets have sufficiently low mass to be detectable at a reasonably high rate. ${ }^{34,35}$

Two experiments that are currently underway will reach sensitivities that would provide a definitive strangelet detection or rule out a significant part of parameter space.

AMS-02: The Alpha Magnetic Spectrometer (AMS) is a space-based particle physics experiment involving several hundred physicists from more than 50 institutions in 16 countries, led by Samuel Ting of MIT. A prototype (AMS-01) flew in June 1998 aboard the Space Shuttle Discovery, ${ }^{36}$ and AMS-02 is currently scheduled to fly to the International Space Station (ISS) in 2008. Once on the ISS AMS-02 will remain active for at least three years. Equipped with a superconducting magnet, time-of-flight detectors, trackers, calorimeter, a ring imaging Cerenkov counter, etcetera, AMS-02 will provide data with unprecedented accuracy on cosmic ray electrons, positrons, protons, nuclei, anti-nuclei and gammas in the GV-TV range and probe issues such as antimatter, dark matter, cosmic ray formation and propagation. In addition it will be uniquely suited to discover strangelets characterized by extreme rigidities for a given velocity compared to nuclei. ${ }^{9,10}$ AMS-02 will have excellent charge resolution up to $Z \approx 26$, and should be able to probe a large mass range for strangelets. A reanalysis of data from the 1998 AMS-01 mission has given hints of some interesting events, such as one with $Z=2, A=16^{37}$ and another with $Z=8$, but with the larger AMS-02 detector running for 3 years or more, real statistics is achievable.

LSSS: The Lunar Soil Strangelet Search (LSSS) is a search for $Z=8$ strangelets using the tandem accelerator at the Wright Nuclear Structure Laboratory at Yale. 
The experiment involves a dozen people from Yale, MIT, and Århus, led by Jack Sandweiss of Yale and studies a sample of 15 grams of lunar soil from Apollo 11. It will reach a sensitivity of $10^{-17}$ over a wide mass range, ${ }^{38,39}$ sufficient to provide detection according to the estimates in Ref. 28 if strangelets have been trapped in the lunar surface layer. In contrast to the deep oceanic and geological mixing on Earth, the effective mixing depth of the lunar surface is only around one meter, determined largely by micrometeorite impacts. Therefore the surface layer has an effective cosmic ray exposure time of around 500 million years. Combined with the lack of a shielding magnetosphere, this results in an expected strangelet concentration in lunar soil which is four orders of magnitude larger than the concentration on Earth, where the search for strangelets so far has resulted in upper limits only.

\section{Strangelets as ultra-high energy cosmic rays}

Strangelets may even provide an explanation for one of the most interesting mysteries in cosmic ray physics: The existence of cosmic rays with energies well beyond $10^{19} \mathrm{eV}$, with measured energies as high as $3 \times 10^{20} \mathrm{eV} \cdot{ }^{40,41}$ It is almost impossible to find a mechanism to accelerate cosmic rays to these energies. Furthermore ultra-high energy cosmic rays lose energy in interactions with cosmic microwave background photons, and only cosmic rays from nearby (unidentified) sources would reach us with the energies measured. Strangelets circumvent both problems, and therefore provide a possible mechanism for cosmic rays beyond the socalled Greisen-ZatsepinKuzmin (GZK) cutoff: ${ }^{42}$

Acceleration: All astrophysical "accelerators" involve electromagnetic fields, and the maximal energy of a charged particle is proportional to its charge. The charge of massive strangelets has no upper bound in contrast to nuclei, so highly charged strangelets are capable of reaching energies much higher than those of cosmic ray protons or nuclei using the same "accelerator". ${ }^{42}$

The GZK-cutoff is a consequence of ultrarelativistic cosmic rays hitting a $2.7 \mathrm{~K}$ background photon with a Lorentz-factor $\gamma$ large enough to boost the $7 \times 10^{-4} \mathrm{eV}$ photon to energies beyond the threshold of energy loss processes, such as photopion production or photo-disintegration. The threshold for such a process has a fixed energy, $E_{\mathrm{Thr}}$, in the frame of the cosmic ray, e.g., $E_{\mathrm{Thr}} \approx 10 \mathrm{MeV}$ for photodisintegration of a nucleus or a strangelet, corresponding to $\gamma_{\mathrm{Thr}}=E_{\mathrm{Thr}} / E_{2.7 \mathrm{~K}} \approx$ $10^{10}$, or a cosmic ray total energy

$$
E_{\text {Total }}=\gamma_{\mathrm{Thr}} A m_{0} c^{2} \approx 10^{19} A \mathrm{eV}
$$

Since strangelets can have much higher $A$-values than nuclei, this pushes the GZKcutoff energy well beyond the current observational limits for ultra-high energy cosmic rays. ${ }^{42,43}$ 


\section{Conclusion}

Strange quark matter may be absolutely stable in bulk, and smaller lumps (strangelets) can be stable down to some small limiting baryon number. Strangelets may form in a first-order cosmological quark-hadron phase transition (unlikely), or in processes related to ccompact stars (more likely). Flux estimates for lumps reaching our neighborhood of the Galaxy as cosmic rays are in a range that makes it realistic to either detect them in upcoming experiments like AMS-02 or LSSS, or place significant limits on the existence of stable strange quark matter. Should strangelets be discovered it would have profound consequences for our understanding of the strong interactions and for a variety of astrophysical phenomena.

\section{Acknowledgments}

This work was supported by the Danish Natural Science Research Council.

\section{References}

1. J. Madsen, Lect. Notes Phys. 516, 162 (1999).

2. A. R. Bodmer, Phys. Rev. D 41601 (1971).

3. S. A. Chin and A. K. Kerman, Phys. Rev. Lett. 43, 1292 (1979).

4. E. Witten, Phys. Rev. D 30, 272 (1984).

5. E. Farhi and R. L. Jaffe, Phys. Rev. D 30, 2379 (1984).

6. M. G. Alford, K. Rajagopal and F. Wilczek, Phys. Lett. B 422, 247 (1998).

7. R. Rapp, T. Schafer, E. V. Shuryak and M. Velkovsky, Phys. Rev. Lett. 81, 53 (1998).

8. R. Klingenberg, J. Phys. G 27, 475 (2001).

9. J. Sandweiss, J. Phys. G 30, S51 (2004).

10. E. Finch, J. Phys. G 32, S251 (2006).

11. A. Chodos, R. L. Jaffe, K. Johnson, C. B. Thorn and V. F. Weisskopf, Phys. Rev. D 9, $3471(1974)$

12. D. Vasak, W. Greiner, and L. Neise, Phys. Rev. C 34, 1307 (1986).

13. C. Greiner, D.-H. Rischke, H. Stöcker, and P. Koch, Phys. Rev. D 38, 2797 (1988).

14. K. Takahashi and R. N. Boyd, Astrophys. J. 327, 1009 (1988).

15. E. P. Gilson and R. L. Jaffe, Phys. Rev. Lett. 71, 332 (1993).

16. J. Madsen, Phys. Rev. D 50, 3328 (1994).

17. J. Schaffner-Bielich, C. Greiner, A. Diener, and H. Stöcker, Phys. Rev. C 55, 3038 (1997).

18. M. S. Berger and R. L. Jaffe, Phys. Rev. C 35, 213 (1987).

19. J. Madsen, Phys. Rev. Lett. 70, 391 (1993).

20. J. Madsen, Phys. Rev. D 47, 5156 (1993).

21. R. Balian and C. Bloch, Ann. Phys. 60, 401 (1970).

22. H. Heiselberg, Phys. Rev. D 48, 1418 (1993).

23. K. Rajagopal and F. Wilczek, Phys. Rev. Lett. 86, 3492 (2001).

24. J. Madsen, Phys. Rev. Lett. 87, 172003 (2001).

25. J. Madsen, Phys. Rev. Lett. 85, 4687 (2000).

26. V. V. Usov, Phys. Rev. D 70, 067301 (2004).

27. M. Stejner and J. Madsen, Phys. Rev. D 72, 123005 (2005).

28. J. Madsen, Phys. Rev. D 71, 014026 (2005).

29. J. Madsen, Phys. Rev. Lett. 61, 2909 (1988). 
30. J. L. Friedman and R. R. Caldwell, Phys. Lett. B 264, 143 (1991).

31. O. G. Benvenuto and J. E. Horvath, Mod. Phys. Lett. A 4, 1085 (1989).

32. G. A. Medina-Tanco and J. E. Horvath, Astrophys. J. 464, 354 (1996).

33. K. S. Cheng and V. V. Usov, Phys. Rev. D 74, 127303 (2006).

34. J. Madsen, J. Phys. G 28, 1737 (2002).

35. P. Jaikumar, S. Reddy and A. W. Steiner, Phys. Rev. Lett. 96, 041101 (2006); M. G. Alford, K. Rajagopal, S. Reddy and A. W. Steiner, Phys. Rev. D 73, 114016 (2006).

36. M. Aguilar et al. [AMS Collaboration], Phys. Rept. 366, 331 (2002) [Erratum-ibid. 380, 97 (2003)].

37. V. Choutko, in 28th International Cosmic Ray Conference (Universal Academy Press, 2003) 1765.

38. K. Han, J. Phys. G 32, S465 (2006).

39. B. Monreal, nucl-ex/0506012.

40. K. Greisen, Phys. Rev. Lett. 16, 748 (1966).

41. G. T. Zatsepin and V. A. Kuzmin, JETP Lett. 4, 78 (1966) [Pisma Zh. Eksp. Teor. Fiz. 4, 114 (1966)].

42. J. Madsen and J. M. Larsen, Phys. Rev. Lett. 90, 121102 (2003).

43. M. Rybczynski, Z. Wlodarczyk and G. Wilk, Acta Phys. Polon. B 33, 277 (2002). 